\title{
BMC Medical Education reviewer acknowledgement 2015
}

\author{
Clare Partridge
}

\section{Contributing reviewers}

The editors of BMC Medical Education would like to thank all our reviewers who have contributed to the journal in Volume 15 (2015).

\author{
Aase Aamland \\ Norway \\ Rajib Acharya \\ India \\ Wilco Achterberg \\ Netherlands
}

Michelle Aebersold

USA

Akisibadek Afoko

Ghana

Nelia Afonso

USA

Pooja Agarwal

USA

Rola Ajjawi

UK

Fares Alahdab

USA

Najlaa Alamoudi

Saudi Arabia

John Albarran

UK

Mohamed Al-Eraky

Saudi Arabia
Heather Alexander

Australia

Carla Alexander

USA

Syeda Ali

Pakistan

Hanan Al-Kadri

Saudi Arabia

Jill Allison

Canada

Patricia Alpert

USA

Abdullah Alzahem

Saudi Arabia

Anthony Amalba

Ghana

Santushi Amarasuriya

Sri Lanka

Gowri Anandarajah

USA

Douglas Ander

USA

Liz Anderson

UK

\author{
Geoffrey Anderson \\ USA \\ Don Anderson \\ USA \\ Vivienne Anderson \\ New Zealand \\ Allen Andrade \\ USA
}

Matthias Angstwurm

Germany

Nigel Armfield

Australia

Richard Arnett

Ireland

Andleeb Arshad

Kuwait

Elpida Artemiou

Saint Kitts And Nevis

Aimee Aubeeluck

UK

Marc Auerbach

USA

Myo Nyein Aung

Thailand

Correspondence: clare.partridge@biomedcentral.com

BioMed Central, Floor 6, 236 Gray's Inn Road, London WC1X 8HB, UK 
Samy Azer

Saudi Arabia

Maria Manuel Azevedo

Portugal

Warwick Bagg

New Zealand

Laila Bahammam

Saudi Arabia

David Bahner

USA

Yun Bai

China

Lubna Baig

Pakistan

Paul Baker

UK

Constance Baldwin

USA

I Bank

Netherlands

Carlos Baptista

USA

Rebecca Baranowski

USA

Judith Barbaro-Brown

UK

Patrick Barlow

USA

Stephen Barnett

Australia

Deborah Barr

USA

Tony Barrett

New Zealand

Emma Bartle

Australia

Ankur Barua

Nepal

Joanna Bates

Canada

Zeynep Baykan

Turkey

Margaret Bearman

Australia
David Bearn

UK

Stefanie Beck

Germany

Gary Beck

USA

Jan C Becker

Germany

Pierrick Bedouch

France

Deborah Begoray

Canada

Anne Belcher

USA

Mary Bell

Canada

Jochanan Benbassat

Israel

Deirdre Bennett

Ireland

Anne-Marie Bergh

South Africa

Esther Bergman

Netherlands

Robert Bernard

Canada

Allan Bernardo

Macao

Cheri Bethune

Canada

Deepa Bhat

India

Ali Bikmoradi

Iran

Rex Billington

New Zealand

Justin Bilszta

Australia

Adelaide Blavier

Belgium

Julia Blitz

South Africa

Karin Blomberg

Sweden
Daniel Blumenthal

USA

Phil Blyth

New Zealand

Sylvain Boet

Canada

Valdes Bollela

Brazil

Katrien Bombeke

Belgium

Eveline Booij

Netherlands

Thomas Booth

UK

Guido Bortoluzzi

Italy

Hans Martin Bosse

Germany

Joseph Donald Boudreau

Canada

Frances Bourbonnais

Canada

Malcolm Boyle

Australia

Marek Brabec

Czech Republic

Carlos Brailovsky

Canada

Andrei Brateanu

USA

Victoria Brazil

Australia

Jennifer Breckler

USA

Jan Breckwoldt

Switzerland

Gustavo Bregagnollo

Brazil

Jarle Breivik

Norway

Mette Brekke

Norway

David Brinkman

Netherlands 
Eileen Britt

New Zealand

Pier Bryden

Canada

Ryan Brydges

Canada

Alison Bullock

UK

S Bunton

USA

Bryan Burford

UK

M Burge

USA

Annette Burgess

Australia

Julian Burton

UK

Jamiu Busari

Netherlands

Lillian Butungi Niwagaba

USA

Carma Bylund

Qatar

Brian Cameron

Canada

Craig Campbell

Canada

Kendall Campbell

USA

Angela Carberry

Australia

Marian Carey

UK

Jan Carline

USA

Elisabeth Carlson

Sweden

Rosemary Caron

USA

Sandra Carr

Australia

Enrique Castro-Sánchez

UK
Peter Cathro

Australia

Bernard Cerutti

Switzerland

Wen-Lin Chai

Malaysia

Wai Hong Chan

UK

Madawa Chandratilake

Sri Lanka

Shine Chang

USA

Serena Chao

USA

Renata Chapman

Australia

Rodger Charlton

UK

Herbert Chase

USA

Julie Chen

Hong Kong

Yen-Yuan Chen

Taiwan

Jack Chen

USA

Yan Chen

New Zealand

Han-Sun Chiang

Taiwan

Tudor Chinnah

UK

Tobias Chirwa

South Africa

Margaret Chisolm

USA

Yen-Lin Chiu

Taiwan

Jane Chueh

USA

Wanicha Chuenkongkaew

Thailand

Anna Chur-Hansen

Australia
Giancarlo Cicolini

Italy

Jeannie Cimiotti

USA

Sarah Clark

USA

Kevin Clauson

USA

Jennifer Cleland

UK

Lynn Clouder

UK

David Coady

UK

Deborah Cohen

UK

James Colbert

USA

Hoffie Conradie

South Africa

Ronan Conroy

Ireland

Siobhan Cooke

UK

Simon Cooper

Australia

Marie Corkery

USA

Manuel Costa

Portugal

Ian Couper

South Africa

Alison Cowley

UK

Gregory Crawford

Australia

Caroline Crawford

USA

Hilary Creed-Kanashiro

Peru

Jim Crossley

UK

Sylvia Cruess

Canada 
Laura Cullen

USA

Sally Curtis

UK

Fiona Curtis

UK

Tara Cusack

Ireland

Eugene Custers

Netherlands

Michele Daly

Australia

Kay Daniels

USA

Mary Dankbaar

Netherlands

Jeanette Dawa

Kenya

Andrew Day

Australia

AJ de Beaufort

Netherlands

Walter De Caro

Italy

Elizabeth Dean

Canada

Clare Delany

Australia

Dianne Delva

Canada

Marcel D'Eon

Canada

Luca Dessy

Italy

Shafik Dharamsi

Canada

Joost Dijkstra

Netherlands

Konstantinos Dimitriadis

Germany

Janine Dizon

Australia

Mamuka Djibuti

Georgia
Sarah Dobson

Canada

Eva Doherty

Ireland

Anthony Donato

USA

Chaoyan Dong

Singapore

Peter Donkor

Ghana

Lachlan Doughney

Australia

Athanassios Douzenis

Greece

Alan Dow

USA

John Roger Downie

UK

Aoife Doyle

UK

Brian Drolet

USA

Robbert Duvivier

Netherlands

Craig Eberson

USA

Samuel Edelbring

Sweden

Gudrun Edgren

Sweden

Daniel Edwards

Australia

Jan Ehlers

Germany

Diann Eley

Australia

Rodrigo Elizondo Omaña

Mexico

Simone Elliott

Australia

Matthew Ellis

UK

Amal El-Moamly

Egypt
Adel Elmoselhi

United Arab Emirates

Ruth Endacott

UK

Rebecca Erschens

Germany

Francisco Escobar-Rabadán

Spain

Begoña Espejo

Spain

Goetz Fabry

Germany

Liz Falconer

UK

Angela Fan

Taiwan

Najat Farsi

Saudi Arabia

David Feldstein

USA

Li Felländer-Tsai

Sweden

Rubens Fernandes

Brazil

Laura Fieschi

Italy

James Fisher

UK

James Fitzgerald

UK

Jonathan Flacker

USA

Margot Fleuren

Netherlands

Maaike Flinkenflögel

Rwanda

Signe Flottorp

Norway

Cornelia Fluit

Netherlands

Eleanor Flynn

Australia

Kirsty Foster

Australia 
Janneke Frambach

Netherlands

Chris Frampton

New Zealand

Zeno Franco

USA

Brandi Freeman

USA

Adrian Freeman

UK

Garth Funston

UK

Daniel Furmedge

UK

Sonya Gabrielian

USA

Robert Gagnon

Canada

Peter Gallagher

Nigeria

Jenny Gallagher

UK

Gisselle Gallego

Australia

Hong Gao

USA

Ana Garces

Guatemala

Jayne Garner

UK

Mark Garside

UK

Fasika Amdeslasie Gebrekirkos

Ethiopia

David Gijbels

UK

Kirsty Gillgrass

UK

Ricci Giovanna

Italy

Nadine Göb

Germany

Joanne Goldman

Canada
Kenneth Goldschneider

USA

Melody Goodman

USA

Adam Gordon

UK

Chivaugn Gordon

South Africa

Deepthiman Gowda

USA

Gertraud Gradl

Germany

Jasnet Grant

UK

Maria Caterina Grassi

Italy

Andy Gray

South Africa

Ben Gray

New Zealand

Page Gregory

UK

Larry Gruppen

USA

Anthony Guerrero

USA

Bruno Guimarães

Portugal

Lisa Guirguis

Canada

Freedom Nkhululeko Gumedze

South Africa

Ronny Gunnarsson

Australia

Geney Gunston

South Africa

Puneet Gupta

India

Susan Guralnick

USA

Michele Haight

USA

Iassen Halatchliyski

Germany
Suraya Hamid

Malaysia

John Hamilton

Australia

Dana Hammer

USA

Wolfgang Hampe

Germany

Heeyoung Han

USA

Ailish Hannigan

Ireland

Cynthia Haq

USA

Eugene Harris

USA

Reema Harrison

Australia

Christopher Harrison

UK

James Harrison

USA

Daniel Hashimoto

USA

Andrew Hassell

UK

Rosemarie Hatala

Canada

Kamila Hawthorne

UK

Richard Hays

Australia

Iman Hegazi

Australia

Elizabeth Hemphill

Australia

Jeroen Hendriks

Australia

Marcus Henning

New Zealand

Mark Hernandez

Ecuador

Leonie Heskin

Ireland 
Kathleen Hickey

USA

Andrew Hill

New Zealand

Ming-Jung Ho

Taiwan

Yvonne Hodgson

Australia

David Hogan

Canada

Are Holen

Norway

Daniel Holt

USA

Matthias Holzer

Germany

Matt Homer

UK

Jianlin Hou

China

Wendy $\mathrm{Hu}$

Australia

Chin-Chou Huang

Taiwan

Judith Hudson

Australia

Nicky Hudson

Australia

Christine Hughes

Canada

Daniel Huhn

Germany

Steinar Hunskaar

Norway

Fawzia Huq

USA

Sören Huwendiek

Switzerland

Nahla Ibrahim

Saudi Arabia

December Ikah

UK

Dragan Ilic

Australia
Rintaro Imafuku

Japan

Kevin Imrie

Canada

Gerard Ingham

Australia

Farhana Irfan

Saudi Arabia

Fabian Jacobs

Germany

Mohammad Jalili

Iran

Saroj Jayasinghe

Sri Lanka

Anthony Jerant

USA

Diane Jette

USA

Jun Jin

Hong Kong

Maree Johnson

Australia

Carolyn Johnston

UK

Diana Jonas-Dwyer

Australia

Michael Jones

Australia

Melvyn Jones

UK

Therese Jones

USA

Christine Jorm

Australia

Tanisha Jowsey

New Zealand

Noelle Junod Perron

Switzerland

Haytham Kaafarani

USA

Amudha Kadirvelu

Malaysia

Martina Kadmon

Germany
Therese Kairuz

Australia

Lisa Kane Low

USA

Maher Karam

USA

Andre Karger

Germany

Syed Irfan Karim

Saudi Arabia

Indika Karunathilake

Sri Lanka

Adetayo Kasim

UK

Aliya Kassam

Canada

Anne Kawamura

Canada

Yang Ke

China

Melanie Keep

Australia

Carolina Keijsers

Netherlands

Michelle Kelly

Australia

Martina Kelly

Canada

Amanda Kenny

Australia

Athol Kent

South Africa

Junaid Sarfraz Khan

Pakistan

Umatul Khoiriyah

Indonesia

Jens Folke Kiilgaard

Denmark

James Kilgour

UK

Sharla King

Canada

Anne Kirchhoff

USA 
Irene Betty Kizza

Uganda

Linda Klein

Australia

Robert Kleinert

Germany

Petra Knaup

Germany

Richard Knox

UK

Sarah Koenig
Germany

Jennifer Kogan

USA

Jillian Kohler

Canada

Joseph Kolars

USA

Michaela Kolbe

Switzerland

Jill Konkin

Canada

Andrzej Kononowicz

Sweden

Andries Koster

Netherlands

Klaus Kroencke

Germany

Edward Krupat

USA

Kulamakan Kulasegaram

Canada

Koshila Kumar

Australia

Grace M Kuo

USA

Viji Kurup

USA

Rashmi Kusurkar

Netherlands

Patrick Kyamanywa

Rwanda

Anita Laidlaw

UK
Trevor Lambert

UK

Ekaterini Lambrinou

Cyprus

Andrew Lane

Australia

Vicki Langendyk

Australia

Susan Langmore

USA

Marianna Lanoue

USA

Philip Larkin

Ireland

Jason Last

Ireland

Anandi Law

USA

Elizabeth Lazzara

USA

Heather Leary

USA

Michael Leathley

UK

Nikki Lee

Netherlands

Wui-Chiang Lee

Taiwan

Jennifer Leeman

USA

Janet Lefroy

UK

Ronny Lehmann

Germany

Paolo Leombruni Italy

Jimmie Leppink

Netherlands

Kat Leung

Australia

Linda Lewin

USA

Robin Lewis

UK
Su-Ting Li

USA

Sok Ying Liaw

Singapore

Steven Lillis

New Zealand

Blossom Lin

Taiwan

Michelle Lincoln

Australia

Stefan Lindgren

Sweden

Liliane Lins

Brazil

Kristin Lo

Australia

Jocelyn Lockyer

Canada

Stephen Loftus

USA

Lia Logio

USA

Lawrence Loo

USA

Elizabete Loureiro

Portugal

Alwyn Louw

South Africa

Karen Luetsch

Australia

Robert Michael Lundin

UK

Paul Lyons

USA

Monica Lypson

USA

Marion Maar

Canada

Martin Macdowell

USA

Mohi Eldin Magzoub

Egypt

Takatoshi Makino

Japan 
Marianne Mak-van der Vossen

Netherlands

Bunmi Malau-Aduli

Australia

Neha Malhotra

New Zealand

Stephen Maloney

Australia

Silvia Mamede

Netherlands

Kate Mandeville

UK

Dianne Manning

South Africa

Katherine Margo

USA

Maurice Mars

South Africa

Aaron Marshall

USA

Lene Martin

Sweden

Yousef Marwan

Kuwait

Robert Mash

South Africa

Italo Masiello

Sweden

David Matheson

UK

Edward Matsumoto

Canada

Nikos Mattheos

Hong Kong

Maliza Mawardi

Malaysia

Simon Maxwell

UK

Win May

USA

Miguel Angel Mayer

Spain

Odette Mazel

Australia
Ruth McMenamin

Ireland

Lynn McBain

New Zealand

Lise McCoy

USA

Matthew McGrail

Australia

Deirdre McGrath

UK

Danette McKinley

USA

Michelle McLean

Australia

Chris McManus

UK

Onno Meijer

Netherlands

Ryan Meili

Canada

Stewart Mennin

USA

Sultan Ayoub Meo

Saudi Arabia

Annette Mercer

Australia

Jacqueline Merrill

USA

Neil Merrylees

UK

Jamie Meuser

Canada

Ricarda Mewes

Germany

Barret Michalec

USA

Sandy Middleton

Australia

Brennen Mills

Australia

Taro Minami

USA

Logan Mitchell

New Zealand
Peter Mittwede

USA

Tobias Moczko

Germany

Lise Mogensen

Australia

Mpho Mogodi

Botswana

Victor Mogre

Ghana

Simin Mohebbi

Iran

Andreas Möltner

Germany

Shabir Moosa

South Africa

Meghan Moran

USA

Anne Mette Morcke

Denmark

Prue Morgan

Australia

Priya Morjaria

UK

Christopher P Morley

USA

Alison Mostyn

UK

Christine Moutier

USA

Phillip Mucksavage

USA

Brigitte Mueller-Hilke Germany

Judy Mullan

Australia

Ellen Murgitroyd

UK

Douglas Murphy

UK

Richard Murray

Australia

Deirdre Murray

Ireland 
David Musick

USA

Eduardo Mutto

Argentina

Erisa Mwaka

Uganda

Sun Jung Myung

South Korea

Balakrishnan Nair

Australia

Devaki Nambiar

India

Susan Nancarrow

Australia

Abedini Nauzley

USA

Hamde Nazar

UK

David Ndetei

Kenya

Maarit Nevalainen

Finland

Anne Nevgi

Finland

Jonathan Newbury

Australia

Khuen Yen Ng

Malaysia

Sandra Nicholson

UK

Christoph Nikendei

Germany

Kirstin Nillesen

Netherlands

Somashekhar Nimbalkar India

Gillian Nisbet

Australia

Lorraine Noble

UK

Jonas Nordquist

Sweden

Birgitte Nørgaard

Denmark
Ross Norman

Canada

Zineb Nouns

Switzerland

Bridget Obrien

USA

Sabine Oertelt-Prigione

Germany

Bodil Ohlsson

Sweden

E Oluwabunmi Olapade-Olaopa

Nigeria

Patricia Oliveira

Brazil

Asela Olupeliyawa

Sri Lanka

Hirotaka Onishi

Japan

Amy Opalek

USA

Nancy Oriol

USA

Helen O'Sullivan

UK

Anna Oswald

Canada

Colm O'Tuathaigh

Ireland

Odile Ouwe Missi Oukem-Boyer

Cameroon

Claudio Owino

Kenya

Gozde Ozakinci

UK

Jorge Pales

Spain

Alvisa Palese

Italy

Edward Palmer

Australia

Mariusz Panczyk

Poland

Andrew Papanikitas

UK
Yoon Soo Park

USA

Helena Paro

Brazil

Janet Parsons

Canada

Vimmi Passi

UK

Joanne Pattinson

UK

Andrew Timothy Pattison

UK

David Paul

Australia

Karl Payne

UK

Ian Pearce

UK

Jacob Pearce

Australia

Jon Penm

USA

Pamela Pennington

Guatemala

Jason Perepelkin

Canada

Adam Persky

USA

John Peteet

USA

Harm Peters

Germany

Ray Peterson

Australia

Robin Pettit

USA

Julie Phillips

USA

Jobeth Pilcher

USA

Ralph Pinnock

New Zealand

Jason Pole

Canada 
Susan Pollart

USA

David Ponka

Canada

Sari Ponzer

Sweden

Phillippa Poole

New Zealand

Cornelis Postma

Netherlands

Cheryl Poth

Canada

Merce Prat-Sala

UK

Gill Price

UK

Titi Savitri Prihatiningsih

Indonesia

Amy Prunuske

USA

Ian Puddey

Australia

Livia Puljak

Croatia

Martin Pusic

USA

Raghunath Puttaiah

USA

Louise Racine

Canada

Jan Radford

Australia

Natalie Radomski

Australia

Malathi Raghavan

Canada

Yinusa Raji

Nigeria

Vijaykumar Rajput

USA

Christopher Ramnanan

Canada

Fiza Rashid-Doubell

Bahrain
Tobias Raupach

Germany

Robin Ray

Australia

Mark Raymond

USA

Szabo Rebecca

Australia

John Rees

UK

Paul Rega

USA

Krishna Regmi

UK

Rehana Rehman

Pakistan

Kate Reid

Australia

Marcel Reinders

Netherlands

Shmuel Reis

Israel

L Reis

Brazil

Daniel Reissmann

Germany

Harold Reiter

Canada

Jeremy Richards

USA

Janet Riddle

USA

Arnoldo Riquelme

Chile

Christopher Roberts

Australia

Katie Robinson

USA

Kem Rogers

Canada

Marcy Rosenbaum

USA

Tara Rosewall

Canada
Shelley Ross

Canada

Linda Ross

Australia

Jens Rothenberger

Switzerland

Imogene Rothnie

Australia

Charlotte Rothwell

UK

Joy Rudland

New Zealand

Miriam Rüsseler

Germany

Clark Russell

UK

Aline Saad

Lebanon

Hazim Sadideen

UK

Ratana Saipanish

Thailand

Eduardo Salas

USA

Helen Salisbury

UK

Murali Sambasivan

Malaysia

Dario Sambunjak

Croatia

Sally Santen

USA

Marina Sawdon

UK

Ana Sergio Da Silva UK

Stefan K Schauber

Germany

Noah Schenkman

USA

Sarah Schiekirka

Germany

Carolin Schmid

Germany 
Ralf Schmidmaier

Germany

Kai Philipp Schnabel

Switzerland

Francine Schneider

Netherlands

Andreas Schröder

Denmark

Anne-Mette Schroll

Denmark

Jobst-Hendrik Schultz

Germany

Daniel Schumacher

USA

Katrin Schüttpelz-Brauns

Germany

Karen Scott

USA

Jane Seale

UK

Dean Seehusen

USA

Ahsan Sethi

UK

Saran Shantikumar

UK

John Shaw

New Zealand

Dale Sheehan

New Zealand

Emma Sherry

Australia

Duncan Shrewsbury

UK

Boaz Shulruf

Australia

Matt Sibbald

Canada

D Robert Siemens

Canada

Si Mui Sim

Malaysia

Anne Simmenroth-Nayda

Germany
Christopher Simon

Canada

Gaiane Simonia

Georgia

Scot Simpson

Canada

Vicki Simpson

USA

Veena Singaram

South Africa

Kuldeep Singh

Nepal

Katrin Singler

Germany

Tony Skapetis

Australia

Ruth Sladek

Australia

Helen Slater

Australia

Lars Småbrekke

Norway

Sydney Smee

Canada

Claire Smith

UK

Robert Smith

USA

Stefanus Snyman

South Africa

Ingunn Bjarnadottir Solberg

Norway

Zdenko Sonicki

Croatia

Penny Standen

USA

Karen Stegers-Jager

Netherlands

Jost Steinhäuser

Germany

Aslak Steinsbekk

Norway

Fred Stevens

Netherlands
Michael Stevenson

UK

Lyn Stewart

Australia

Rosalyn Walker Stewart

USA

Fredrich Stiefel

Switzerland

Philipp Stieger

Germany

Alasdair Strachan

UK

Judith Strawbridge

Ireland

Goran Strkalj

Australia

Alison Sturrock

UK

Virpi Sulosaari

Finland

Samantha Sundercombe

Australia

Tarja Suominen

Finland

Premala Sureshkumar

Australia

Laura Surmon

Australia

Jeannette L Swaan

Netherlands

Meenakshi Swamy

UK

Nur Afrainin Syah

Indonesia

Shima Tabatabai

Iran

Masami Tagawa

Japan

Kenzo Takahashi

Japan

Zohray Talib

USA

Marie Tarrant

Hong Kong 
Ara Tekian

USA

Conor Teljeur

Ireland

Olle ten Cate

Netherlands

Pim Teunissen

Netherlands

Nai-Peng Tey

Malaysia

Axel Themmen

Netherlands

Kassiani Theodoraki

Greece

Johan Thor

Sweden

Sean Tierney

Ireland

Paul Tiffin

UK

Julie K Tilson

USA

Vivienne Tippett

Australia

Jonathon Tomlinson

UK

Jane Toms

UK

William Tormey

Ireland

Deborah Tregunno

Canada

Marc Triola

USA

Shih-Li Tsai

Taiwan

Ilene Tsui

USA

Sharon Tucker

USA

Mike Tweed

New Zealand

Reidar Tyssen

Norway
Jinan Usta

Lebanon

Sjoukje van den Broek

Netherlands

Cees van der Vleuten

Netherlands

Nynke van Dijk

Netherlands

Tamara van Gog

Netherlands

Clare van Hamel

UK

Daniël van Nijlen

Belgium

Dirk van Rooy

Australia

Gerrit van Schalkwyk

USA

Susan van Schalkwyk

South Africa

Chris van Weel

Australia

Marta van Zanten

USA

Cristina Vargas

Spain

Gary Velan

Australia

Joan Vilalta-Franch

Spain

Laura Villa-Torres

USA

Claudio Violato

USA Minor Outlying Islands

Michelle Visser

USA

Elsa Vitale

Italy

Pirashanthie Vivekananda-Schmidt

UK

Carl von Baeyer

Canada

Rukhsana W Zuberi

Pakistan
Judy Wakeling

UK

Gary Walco

USA

Rachel Walker

Australia

Merrilyn Walton

Australia

Jennifer Walton

Canada

Helena Ward

Australia

Terri Warholak

USA

Justin Waring

UK

Simon Watmough

UK

Andy Wearn

New Zealand

Katie Webb

UK

Jennifer Weller

New Zealand

Olwyn Westwood

UK

Sue Whittle

UK

Kristin Wiisanen Weitzel

USA

Marjo Wijnen-Meijer

Netherlands

Deana Wilbanks

USA

Kerry Wilbur

Qatar

Christine Wilder

USA

Margaretha Wilhelmsson

Sweden

Jill Wilkinson

New Zealand

Ingrid Willaing

Denmark 
Lawrence Williams

USA

Graham Williamson

UK

Kathleen Williamson

USA

Deanna Willis

USA

Ian Wilson

Australia

Adam Wilson

USA

Khin Than Win

Australia

Abigail Winkel

USA

Erin Winstanley

USA

Connie Wiskin

UK

Ulrich Woermann

Switzerland

Zane Wolf

USA

Yut Lin Wong

Malaysia
Raymond Wong

USA

Timothy Wood

USA

Robyn Woodward-Kron

Australia

Bjarne Worm

Denmark

Kei Yamada

USA

Che-Ming Yang

Taiwan

Yanqi Yang

Hong Kong

Ahmed Yaqinuddin

Pakistan

Kwang Chien Yee

Australia

Liuhua Ying

China

James Youdas

USA

Greg Young

USA

Omar Young

USA
Tzu-Chieh Yu

New Zealand

Steven Yule

USA

Zamros Yusof

Malaysia

Nikki Zaidi

USA

Benjamin Zendejas

USA

Melvyn Zhang

Singapore

Zhao-Hua Zhong

UK

Stefan Zimmermann

Germany

Sa'ed Zyoud

Palestinian Territory

Aleksander Zywot

Grenada

Published online: 09 February 2016 